\title{
A Study on Target Detection using Covariance Correlation Matrix of Spatial Adaptive Processing
}

Kwan-HyeongLee ${ }^{1}$

${ }^{1}$ Division of Human IT Convergence, DaejinUniv.,POCHEON, Republic of Korea khlee@ daejin.ac.kr

Article History: Received: 11 January 2021; Accepted: 27 February 2021; Published online: 5 April 2021

\begin{abstract}
In this paper, we study for direction of arrival estimation of the desired target in spatial adaptive processing system. The interference signal removed by using the optimal weight of the covariance correlation matrix in order to estimate desired target signal. The spatial adaptive processing system updates the weight of the direction of arrival algorithm to estimate the desired signal. The weight update use an adaptive algorithm such as MUSIC. The optimal weight is obtained by Lagrange multiplier and the covariance correlation matrix. The covariance correlation matrix applies signal phase matching and uses the output power spectrum of the direct of arrival algorithm to estimate the desired target direction. We compare the performance of the proposed method with the existing method by computer simulation. The existing method has poor resolution due to phase errors of $5^{\circ}$ and $-3^{\circ}$ in the estimation of three targets $\left[10^{\circ}, 20^{\circ}, 30^{\circ}\right]$. While, the method proposed in this study accurately estimated the desired three targets. This study proved that the proposed method is superior to the existing method as a result simulation result.
\end{abstract}

Keywords: Direction of arrival, estimation, covariance, correlation matrix, weight.

\section{Introduction}

It have been studied o remove the interference signal. The interference signal removal method has been studied with antenna design method to remove low elevation signal and filtering and signal processing technology to attenuate the power of interference signal [1-2]. The method for effectively removing the interference signal can increase the signal-to-interference noise ratio by using the adaptive array antenna technology [3-4]. Direction of arrival (DoA) estimation method is to find a desired target by removing the interference signal. The target estimation method is an important method to find a desired signal among signals incident on a receiving antenna by radiating a signal through the antenna to the spatial. The direction of arrival estimation method use to estimate for the desired signal in the various research such as radar, sonar, and seismology signal technology. An algorithm for resolving improvement of the direction of arrival estimation has been being developed. The direction of arrival technique estimates the components of the signal by analysis the phase and signal amplitude of the received signal from the antenna [5].

The spatial adaptive processing (SAP) method for noise reduction and power minimization can remove narrow band interference signals and does not cause frequency dependent phase distortion for spread spectrum bandwidthsignal [6-7-15]. A temporary filtering technique is required in signal processing in order to remove a large interference signal such as broad band signal. A spatial only beamforming technique without the adaptive spatial technology can be used by increasing the signal to noise ratio to estimate a desired signal [8-14]. However, this technique increase a side lobe level while increase the signal to noise ratio to find the desired signal. It is sensitive to the timing and distortion errors of the antenna and analogy front-end. An adaptive array antenna with the maximum gain can find the information of the received signal using the optimum weight. The array gain can acquire the maximum gain signal in a desired direction, but the ability to remove interference signal is reduced when the side lobe and null point are not controlled. The spatial adaptive processing can control the phase and gain with each array element of the array antenna.

We propose the desired signal detection of the target using a covariance correlation matrix of spatial adaptive processing. The method of this study proposes the optimal weight using the covariance correlation matrix of spatial adaptive processing. The optimal weight is applied to algorithm in the direction of arrival to detect the desired target. The direction of arrival detection algorithm uses the MUSIC with excellent resolution [9-10]. We compare the performance of the proposed algorithm with the existing algorithm through computer simulation.

The composition of this paper is as follow. Section $2 \mathrm{~d}$ describes the covariance matrix of phase, and Section 3 proposes a correction covariance matrix. Section 4 explore the performance of the proposed algorithm, and closes the paper in section 5 .

\section{Spatial Adaptive Processing}

The spatial adaptive processing generates null for the interference signal with the antenna gain. The signal processing method. In the signal processing method, a signal lager reference signal is regarded as an interference signal. The signal generates a null by applying the weight. The output of each antenna array element in the spatial adaptive processing method is delayed through a tap delay and each tap is multiplied by a complex weight 
to finally produce one output signal. The spatial adaptive processing method is effective in the removing interference signal by applying weight to the receiver. The weight dimension is determined by the number of antenna array and the number of tap delay of each array element [11].

Figure 1 shows the spatial adaptive processing system. The spatial adaptive processing system consists of an array antenna, weight, and tap delay. The array antenna applies linear array and the weight uses the MUSIC arrival direction estimation algorithm. The received signal of each antenna is classified using the tap delay. The signal that have passed the tap delay are multiplied by weight and then summed with the adjacent signals [12]. The spatial adaptive processing array outputs one signal after sum the output of each array element. The signal incident on the array antenna in the Figure 1 can be as follows:

$$
\mathrm{x}(\mathrm{t})=\mathrm{s}(\mathrm{t})+N(t)
$$

The each array element is received k signals. Here, $\mathrm{s}(\mathrm{t})$ and $N(t)$ are the source signal and the noise by the array antenna. The number of antenna arrays 1 to $\mathrm{N}$, and there are $\mathrm{K}$ weights. The $\mathrm{m}$-th array element output is as follows:

$$
\mathrm{x}_{\mathrm{m}}(\mathrm{t})=\sum_{\mathrm{m}=1}^{\mathrm{L}} \mathrm{a}\left(\theta_{\mathrm{m}}\right) \mathrm{s}_{\mathrm{m}}(\mathrm{t})+\mathrm{N}_{\mathrm{m}}(\mathrm{t})
$$

Here, $\mathrm{a}\left(\theta_{\mathrm{m}}\right)$ represents the phase of the target as a steering vector $(\mathrm{m}=1,2, \cdots, \mathrm{L})$. The output of the $\mathrm{m}$-th array element antenna after applying the tap-delay by the equation (2) can be written as follows:

$$
\mathrm{x}_{\mathrm{m}}(\mathrm{t})=\sum_{\mathrm{m}=1}^{\mathrm{L}} \mathrm{C}_{m} \mathrm{a}\left(\theta_{\mathrm{m}}\right) \mathrm{s}_{\mathrm{m}}(\mathrm{t})+\mathrm{N}_{\mathrm{m}}(\mathrm{t})
$$

Here, $\mathrm{C}_{m}$ is a mutual covariance correlation matrix with the parameters multiplied by the weight of the proximity signal after the tap delay. The output of the adaptive array antenna can be expressed as follows in vector format.

$$
\mathrm{x}(\mathrm{t})=\mathrm{CA}(\theta)+N(t)
$$

Here, $A(\theta)=\left[\mathrm{a}\left(\theta_{1}\right), \mathrm{a}\left(\theta_{2}\right), \cdots, \mathrm{a}\left(\theta_{\mathrm{L}}\right)\right]$ is array response vector. A covariance matrix of the array antenna is as follows:

$$
\mathrm{R}=\mathrm{E}\left[\mathrm{x}(\mathrm{t}) \mathrm{x}^{\mathrm{H}}(\mathrm{t})\right]=A \mathrm{R}_{\mathrm{S}} \mathrm{A}^{\mathrm{H}}+\mathrm{R}_{\mathrm{N}}
$$

Here, $R_{S}=E\left[s(t) s^{H}(t)\right]$ is defined asignal covariance matrix, $R_{N}=E\left[N(t) N^{H}(t)\right]$ is a noise covariancematrix, and []$^{H}$ is hermit matrix. The output of the spatial adaptive array considering the weight is as follows:

$$
\mathrm{Y}(\mathrm{t})=\sum_{i=1}^{N} \sum_{m=1}^{L} \sum_{j=1}^{K} W_{j}^{H} X_{i, m}(t)
$$

Here, $\mathrm{W}=\left[\mathrm{w}_{1}, \mathrm{w}_{2}, \cdots, \mathrm{w}_{\mathrm{K}}\right]$ is a weight. The weight of equation (6) apply an adaptive is applied by adaptive array algorithm such as MUSIC and ESPRIT. The interference signal is removed by applying a weight to each array element. The interference removal method delays the signal received by the array antenna in the tap delay. The delayed signal is added to the tap delay signal and the previous signal. The tap delay signal and the previous signal are multiplied by a weight to find a correlation. The desiredsignal is closely correlation between the signals, the interference signal has a low correlation. The spatial adaptive processing energy spectrum for output power minimize is as follows:

$$
\mathrm{P}=\mathrm{E}\left[|\mathrm{Y}(\mathrm{t})|^{2}\right]=\mathrm{W}^{\mathrm{H}} \mathrm{R} \mathrm{W}
$$

We find the optimal weight that minimize the output power in order to estimate the desired signal. The optimal weight has the maximum gain and phase for desired signal and null for the interference signal. The weight has a constraint for uniform gain of the received signal. When the source signal is received to the desired direction, the weight constraint is as follows:

$$
\mathrm{W}^{\mathrm{H}} \mathrm{x}(\varnothing)=\mathrm{G}
$$

Here, $\mathrm{g}$ is the gain. The weight constraint of the interference signal is as follows when the interference signal is received to the desired direction.

$$
\mathrm{W}^{\mathrm{H}} \mathrm{x}(\varnothing)=0
$$

The constraint matrix of equation (7) and equation (8) is expressed as follows:

$$
\left[\begin{array}{l}
\mathrm{W}^{\mathrm{H}} \mathrm{x}(\varnothing) \\
\mathrm{W}^{\mathrm{H}} \mathrm{x}(\varnothing)
\end{array}\right] \mathrm{W}^{\mathrm{H}}=\left[\begin{array}{l}
\mathrm{G} \\
0
\end{array}\right] \text { subject to } W^{H} \mathrm{C}=\mathrm{Q}
$$

We apply Lagrange multipliers method to estimate of the optimal weight. We apply the Lagrange multipliers method to equation (7), and can be expressed as follows:

$$
\mathrm{P}=\mathrm{W} \mathrm{RW}^{\mathrm{H}}-\lambda\left[\mathrm{W}^{\mathrm{H}} \mathrm{C}-\mathrm{Q}\right]
$$

Here $\lambda$ is Lagrange multiplier. In order to obtain the optimal weight, equation (11) can be expressed as follow by differentiating the weight in term of both sides. 
The power spectrum of direction of arrival (MUSIC) is as follow:

$$
\begin{aligned}
\frac{\partial P}{\partial W} & =2 R W+\lambda(C-Q)=0 \\
\mathrm{~W} & =-\frac{1}{2} \lambda R^{-1}(C-Q)
\end{aligned}
$$

$$
P_{\text {spec }}=\frac{A^{H}(\theta) A(\theta)}{W^{H} A(\theta) W A^{H}(\theta)}
$$

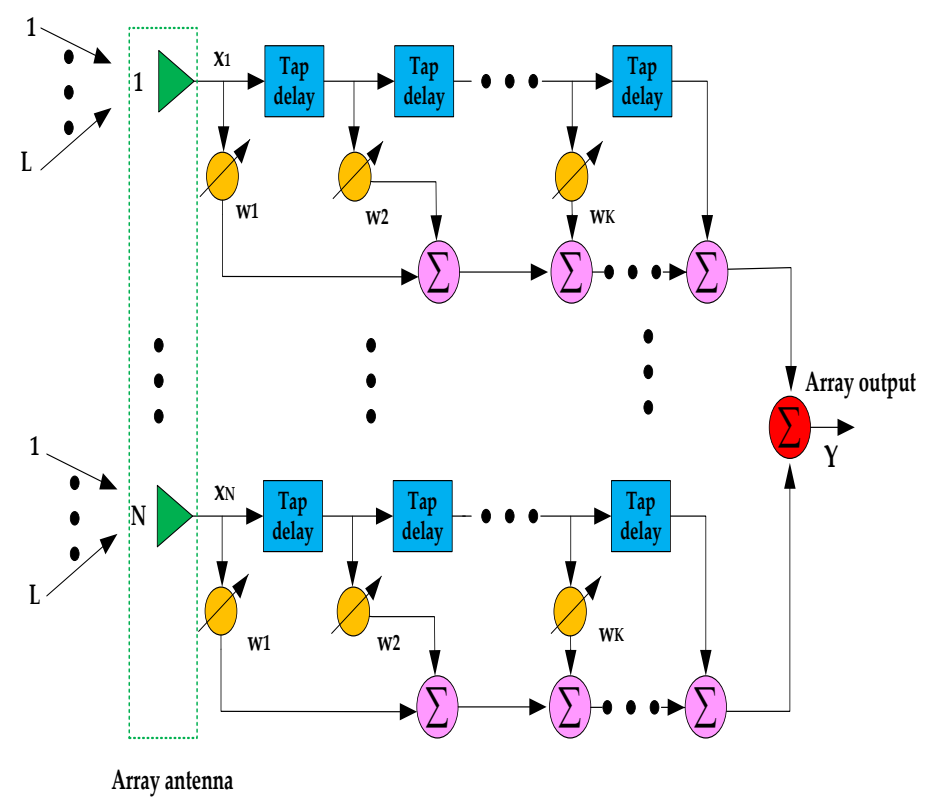

Figure 1. Spatial Adaptive Processing System

\section{Covariance Correlation Matrix Proposal}

This chapter studies covariance correlation matrix to obtain the optimal weight. If the correlation matrix by weight is not correction, the optimal weight cannot be obtained because the covariance values are different. We present the relative of the phase of the each correlation matrix based on the first antenna element to correct correlation matrix [13]. The phase of the covariance correlation matrix is as follow:

$$
\mathrm{C}_{\mathrm{m}}=\left[\begin{array}{c}
1 \\
\mathrm{e}^{\mathrm{j}\left(\phi_{2}-\phi_{1}\right)} \\
\vdots \\
\mathrm{j}^{\mathrm{j}\left(\phi_{\mathrm{L}}-\phi_{\mathrm{L}-1}\right)}
\end{array}\right]=\left[\begin{array}{c}
\mathrm{e}^{\mathrm{j} \phi_{1}} \\
\mathrm{e}^{\mathrm{j} \phi_{2}} \\
\vdots \\
\mathrm{e}^{\mathrm{j} \phi_{\mathrm{L}}}
\end{array}\right]
$$

The equation (5) of correlation matrix is expressed as follows:

$$
\begin{aligned}
& R=E\left[x(t) x(t)^{H}\right] \\
& =E\left[\begin{array}{cccc}
x_{1}(t) x_{1}(t)^{*} x_{1}(t) x_{2}(t)^{*} \cdots & x_{1}(t) x_{L}(t)^{*} \\
x_{2}(t) x_{1}(t)^{*} x_{2}(t) x_{2}(t)^{*} \cdots & x_{2}(t) x_{L}(t)^{*} \\
\vdots & \vdots & \ddots & \vdots \\
x_{L}(t) x_{1}(t)^{*} & x_{L}(t) x_{2}(t)^{*} \cdots & x_{L}(t) x_{L}(t)^{*}
\end{array}\right]
\end{aligned}
$$

Here E[] and ( $)^{*}$ are an expected value and complex conjugate, respectively. Eq. (3) is the covariance matrix without correcting the phase mismatch between channels. To calibrate the covariance matrix, the output signal of the $\mathrm{m}^{\text {th }}$ channel should be divided by the phase mismatch of the $\mathrm{m}^{\text {th }}$ channel. In other words, the gain should be divided and the phase should be subtracted. The corrected signal is as follows:

$$
\mathrm{x}_{\mathrm{m}}^{\mathrm{mc}}(\mathrm{t})=\frac{\mathrm{x}_{\mathrm{m}}(\mathrm{t})}{\exp \left(j \emptyset_{\mathrm{m}}\right)}
$$

The equation (17) can be expressed in vector form

$$
x_{\text {cor }}(t)=\left[x_{1}^{m c}(t), x_{2}^{m c}(t), \cdots, x_{L}^{m c}(t)\right]^{T}
$$

The correction matrix cab be expressed as:

$$
\mathrm{R}_{\text {cor }}=\mathrm{R} \otimes \mathrm{C}_{m}=\mathrm{E}\left[\mathrm{x}_{\text {cor }}(\mathrm{t}) \mathrm{x}_{\text {cor }}(\mathrm{t})^{\mathrm{H}}\right]
$$




$$
\begin{aligned}
& =E\left[\begin{array}{cccc}
\mathrm{x}_{1}^{\mathrm{mc}}(\mathrm{t}) \mathrm{x}_{1}^{\mathrm{mc}}(\mathrm{t})^{*} & \mathrm{x}_{1}^{\mathrm{mc}}(\mathrm{t}) \mathrm{x}_{2}^{\mathrm{mc}}(\mathrm{t})^{*} \cdots & \mathrm{x}_{1}^{\mathrm{mc}}(\mathrm{t}) \mathrm{x}_{\mathrm{L}}^{\mathrm{mc}}(\mathrm{t})^{*} \\
\mathrm{x}_{2}^{\mathrm{mc}}(\mathrm{t}) \mathrm{x}_{1}^{\mathrm{mc}}(\mathrm{t})^{*} & \mathrm{x}_{2}^{\mathrm{mc}}(\mathrm{t}) \mathrm{x}_{2}^{\mathrm{mc}}(\mathrm{t})^{* \cdots} & \mathrm{x}_{2}^{\mathrm{mc}}(\mathrm{t}) \mathrm{x}_{\mathrm{L}}^{\mathrm{mc}}(\mathrm{t})^{*} \\
\vdots & \vdots & \ddots & \vdots \\
\mathrm{x}_{\mathrm{L}}^{\mathrm{mc}}(\mathrm{t}) \mathrm{x}_{1}^{\mathrm{mc}}(\mathrm{t})^{*} & \mathrm{x}_{\mathrm{L}}^{\mathrm{mc}}(\mathrm{t}) \mathrm{x}_{2}^{\mathrm{mc}}(\mathrm{t})^{* \cdots} & \mathrm{x}_{\mathrm{L}}^{\mathrm{mc}}(\mathrm{t}) \mathrm{x}_{\mathrm{L}}^{\mathrm{mc}}(\mathrm{t})^{*}
\end{array}\right]
\end{aligned}
$$

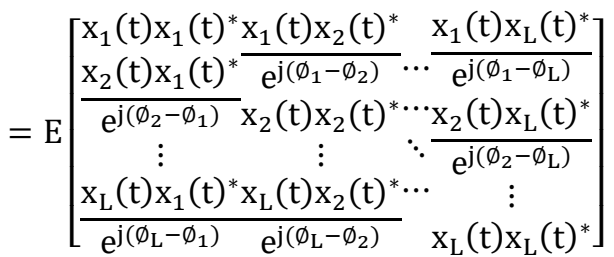

Here the mutual covariance correction matrix can be expressed as:

$$
\mathrm{C}=\left[\begin{array}{cccc}
1 & 1 /\left(\mathrm{e}^{\mathrm{j}\left(\phi_{1}-\phi_{2}\right)}\right) & \cdots & 1 /\left(\mathrm{e}^{\mathrm{j}\left(\phi_{1}-\phi_{\mathrm{L}}\right)}\right) \\
1 /\left(\mathrm{e}^{\mathrm{j}\left(\phi_{2}-\phi_{1}\right)}\right) & 1 & \cdots & 1 /\left(\mathrm{e}^{\mathrm{j}\left(\phi_{2}-\phi_{\mathrm{L}}\right)}\right) \\
\vdots & \vdots & \ddots & \vdots \\
1 /\left(\mathrm{e}^{\mathrm{j}\left(\phi_{\mathrm{L}}-\phi_{1}\right)}\right) & 1 /\left(\mathrm{e}^{\mathrm{j}\left(\phi_{\mathrm{L}}-\phi_{2}\right)}\right) & \cdots & 1
\end{array}\right](22)
$$

\section{Computer Simulation}

This chapter compares and analysis the performance of the proposed method and the existing MUSIC method through simulation. The simulation conditions were set to 9 antenna arrays, a signal-to-noise ratio of $20 \mathrm{~dB}$, and an antenna spacing half wavelength. Figure 2 shows direction of arrival estimation using only MUSIC method for the desired target signal $\left[-5^{\circ}, 0^{\circ}, 5^{\circ}\right]$ in coherent correlation channel. Figure 2 does not estimate the three targets, and the signal estimation resolution is significantly reduced. Figure 3 shows the target estimation by applying the covariance correlation matrix of the spatial adaptive processing to the MUSIC method. Figure 3 accurately detected of the three targets $\left[-5^{\circ}, 0^{\circ}, 5^{\circ}\right]$ to calculate the target estimation error.

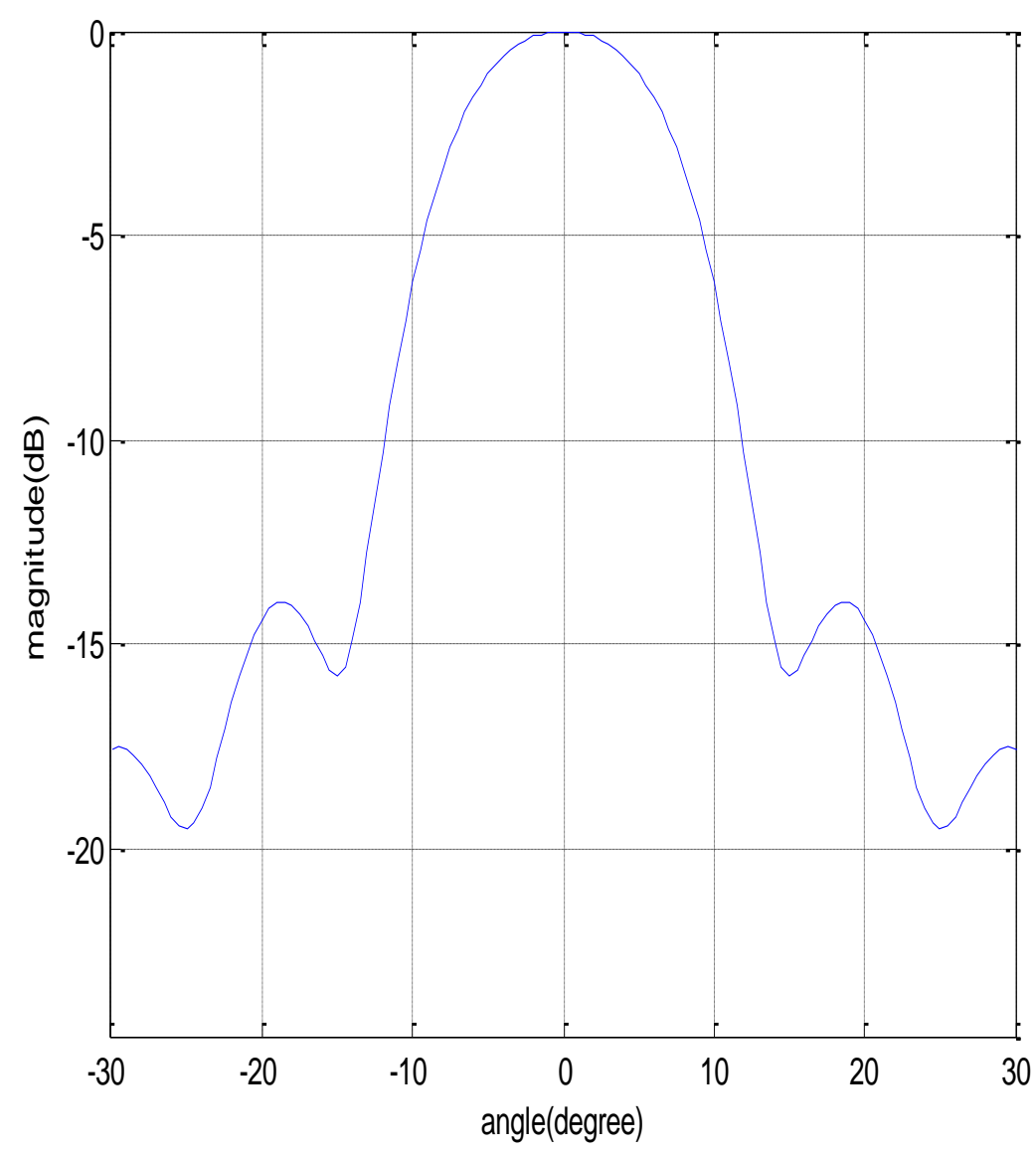

Figure 2. MUSIC DoA at $\left[-5^{\circ}, 0^{\circ}, 5^{\circ}\right]$ 


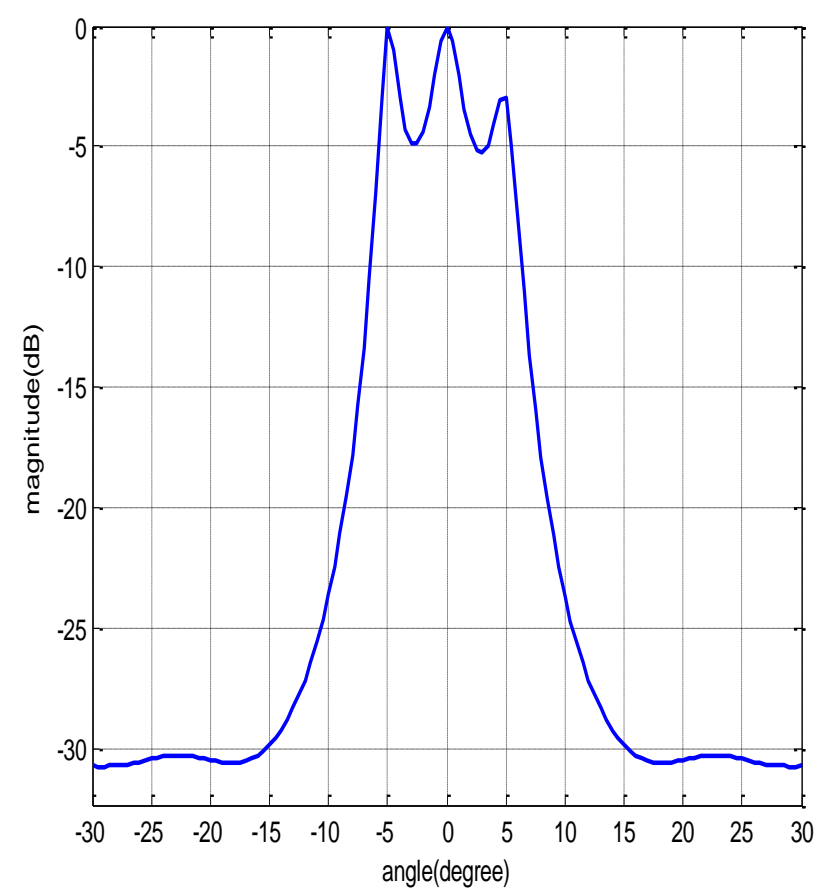

Figure 3. MUSIC DoA in SAP at $\left[-5^{\circ}, 0^{\circ}, 5^{\circ}\right]$

Figure 4 and Figure 5 estimate the desired information signal by increasing the target separation distance. Figure 4 shows the estimation of the direction of arrival using the MUISC method on the desired target $\left[10^{\circ}, 20^{\circ}\right.$, $\left.30^{\circ}\right]$. Although three targets were estimated, target direction of arrival in $20^{\circ}$ and $30^{\circ}$ shows $5^{\circ}$ and $-3^{\circ}$ phase error, respectively. Figure 5 shows the estimation of three target $\left[10^{\circ}, 20^{\circ}, 30^{\circ}\right]$ using the MUSIC method by spatial adaptive processing. Figure 5 accurately estimates the desired three targets.

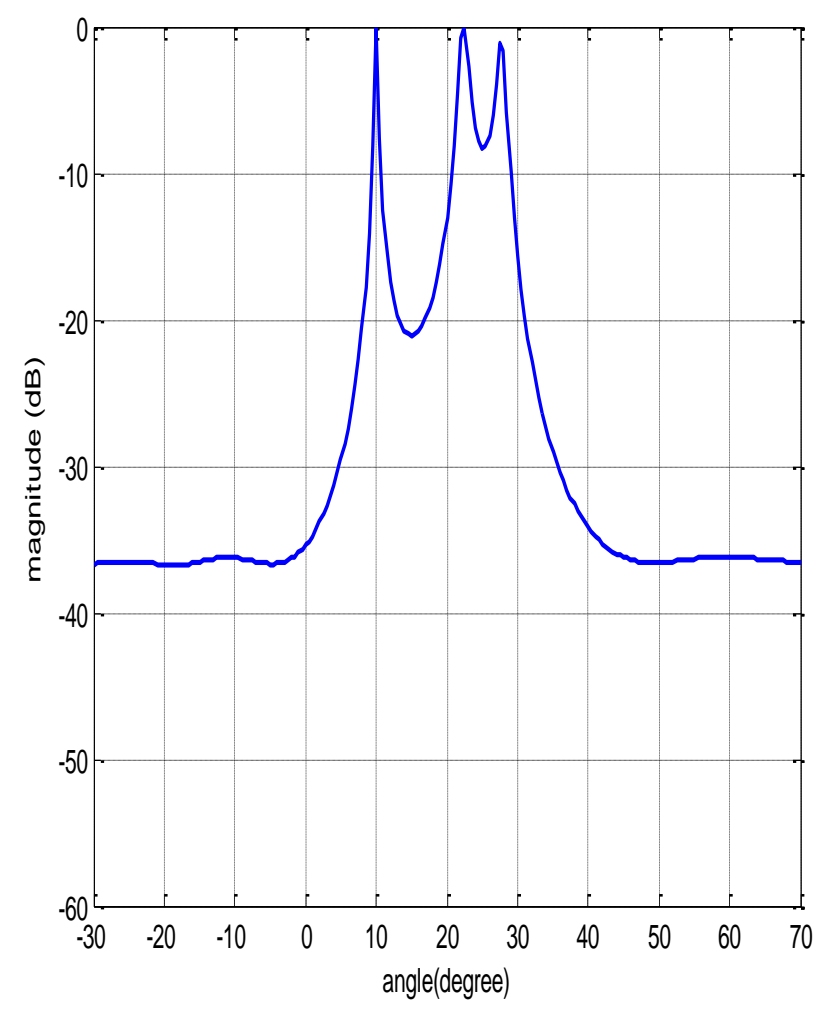

Figure 4. MUSIC DoA estimation 


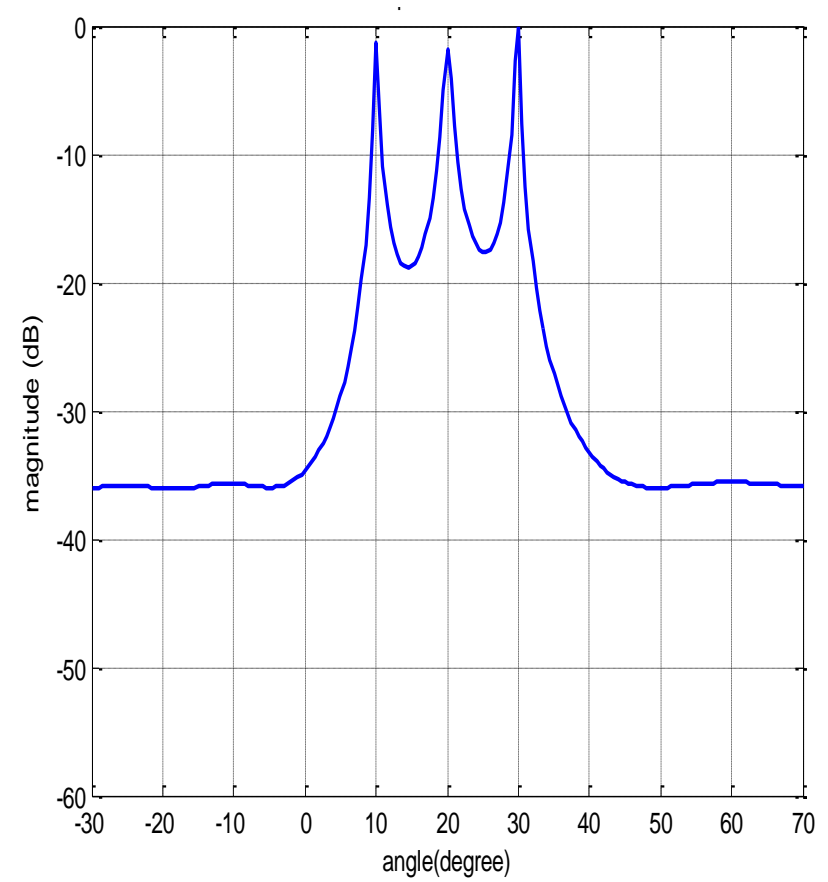

Figure 5. MUSIC DoA estimation with SAP

\section{Conclusion}

In this paper, we studied the optimal weighted covariance correlation matrix to estimate the desired signal. The covariance correlation matrix is updated in weight to remove interference signals using phase matching of the proximity signals. The optimal weight is obtained using the Lagrange multipliers and the covariance correlation matrix and accurately applied to the direct of arrival estimation algorithm to accurately estimate the desired target in the space. We compared the performance of the proposed method with the existing method by simulation. The existing MUSIC algorithm does not accurately estimate the desired target, but the proposed algorithm in this study accurately estimates the desired target. The existing method has poor resolution due to a phase error when estimating the desired target, but the proposed method improves the resolution by estimating exactly three targets. The resolution to estimate the target distance is a very important factor in estimating the direction of arrival. The results of this study proved that the proposed method was superior to the previous method in estimating the direction of arrival and the resolution.

\section{References}

1. Allen, B.; andGhavemi, M.,\& McGraw, Hill. (2005). Adaptive Array system.

2. Li, F.; and Vaccaro, R. J. (1992). Sensitivity of DoA Estimation Algorithm to Sensor Array. IEEE Transactions on Aerospace and Electronic System, 28(3), 708-717.

3. Freidlander, B. (1994). Sensitivity Analysis of the Maximum Likehood Direction Finding Algorithm. IEEE Transactions on Aerospace and Electronic System, 26(6), 953-968.

4. Devalla, Vindhay.; and Prakash. Om. (2014). Development in Unmanned Powered Parachute Aerial Vehicle: A Review. IEEE Transactions on Aerospace and Electronic System Magazine, 29(11), 6-20.

5. Haug, A. J.; and Jacyna. G. M. (2000). Theory and Analytical Performance Evaluation Generalized Correlation. IEEE Journal of Oceanic Engineering, 25(3), 314-330.

6. Wallace, W.; and Michal, A. (2009). Sparse Power Angle Spectrum Estimation. IEEE Transactions and Propagation, 57(8), 2453-2460.

7. Nagata, Yoshifumi,;Fjuioka, Toyate.; and Abe, Masato. (2007). Two-dimensional DoA Estimation of sound Source based on Weighted Wiener Gain Exploiting Two-Directional Microphones. IEEE Transactions on Audio, Speech, and Language Processing, 15(2), 416-429.

8. Han, H. D.; and Ding, Z. (2013) Steepest Descent Algorithm Implementation for Multichannel Blind Signal Recovery. IET communications, 6(18), 3196-3203.

9. Schmidt, R. (2003). Multiple Emitter Location and Signal Parameter Estimation. IEEE Transactions on Antennas and Propagation, 34(9), 1069-1079. 
10. Biha, Le. N.; and Marx, J. I. (2007). MUSIC Algorithm for Vector Sensor Array using Biquaternions. IEEE Transactions on Signal Processing, 55(9), 4523-44533.

11. Lee. K. H. (2019). A Study on the Covariance Matrix Correction of Correlation Signal for Correct Direction Estimation in Phase Mismatch. Lecture Notes in Electrical Engineering, 621(1), 85-90.

12. Vastola, K. (2003). On Robust Wiener Signal Estimation. IEEE Transactions on Automatic Control, $31(5), 466-467$.

13. Lee. K. H. (2018). Signal Estimation using Covariance Matrix of Mutual Coupling and Mean Square Error. The Journal of Korea Institute of Information, Electronics and Communication Technology, 11(6), 691-696.

14. Jović, D. (2018). Accession to the European Union and perception of external actors in the Western Balkans. Croatian International Relations Review, 24(83), 6-32.

15. Kopar, R. (2018). Resource Nationalism in International Investment Law by Sangwani Patrick Ng'ambi. Croatian International Relations Review, 24(83), 125-128. 